\title{
Knockdown of TACC3 inhibits trophoblast cell migration and invasion through the PI3K/Akt signaling pathway
}

\author{
XIAOJUN ZHU, QIANQIAN CAO, XIA LI and ZHENGPING WANG \\ Department of Obstetrics, Women's Hospital, School of Medicine, Zhejiang University, \\ Hangzhou, Zhejiang 310006, P.R. China
}

Received August 7, 2015; Accepted August 1, 2016

DOI: $10.3892 / \mathrm{mmr} .2016 .5659$

\begin{abstract}
The insufficient invasion of trophoblasts is known to be correlated with the development of preeclampsia. Transforming acidic coiled-coil protein 3 (TACC3), a member of the TACC domain family, is important in the regulation of cell differentiation, migration and invasion. However, the role of TACC 3 in trophoblast function during placental development remains to be fully elucidated. The present study aimed to determine the expression and function of TACC 3 in human placenta and to examine the underlying mechanisms. TACC3 expression was analyzed in preeclamptic placentas using reverse transcription-quantitative polymerase chain reaction and western blotting. Cell proliferation was determined by the MTT assay, and cell migration and invasion were measured using Transwell assays. The expression levels of TACC3, matrix metalloproteinase (MMP)-2, MMP-9, tissue inhibitor of metalloproteinase (TIMP)-1, TIMP-2, phosphoinositide 3-kinase (PI3K), phosphorylated (p)-PI3K, AKT and p-AKT were detected by western blotting. The results showed that the expression of TACC 3 was downregulated in preeclamptic placentas. The knockdown of TACC3 significantly inhibited HTR8/SVneo cell proliferation, migration and invasion, and inhibited the expression of matrix metalloproteinases. In addition, the knockdown of TACC 3 significantly reduced the levels of p-PI3K and Akt in the HTR8/SVneo cells. Taken together, the results of the present study demonstrated that the knockdown of TACC 3 inhibited the migration and invasion of HTR8/SVneo cells through suppression of the PI3K/Akt signaling pathway. Therefore, TACC 3 may serve as a novel potential target for treating preeclampsia.
\end{abstract}

Correspondence to: Dr Zhengping Wang, Department of Obstetrics, Women's Hospital, School of Medicine, Zhejiang University, 1 Xueshi Road, Hangzhou, Zhejiang 310006, P.R. China E-mail: wang_zhengping004@163.com

Key words: preeclampsia, transforming acidic coiled-coil protein 3, invasion, matrix metalloproteinases

\section{Introduction}

Preeclampsia is a major disorder of pregnancy and a leading contributor to maternal and perinatal morbidity and mortality rates (1). Although reduced trophoblast proliferation, aberrant trophoblast differentiation, limited migration and invasion of trophoblasts in the uterus, poor remodeling of spiral arteries, and excessive apoptosis have been associated with preeclampsia (2-5), the molecular mechanisms underlying the onset and progression of preeclampsia remain to be fully elucidated, therefore, it is important to investigate these molecular mechanisms of preeclampsia.

The transforming acidic coiled-coil protein (TACC) family is characterized by a conserved C-terminal 'TACC domain' (6). In mammals, three TACC proteins are expressed from three genes, TACC1, TACC2 (also known as AZU-1 and ECTACC) and TACC3 (also known as AINT and ERIC1) (7). During mitosis, the TACC proteins localize around the centrosomes and are important for the organization of microtubule organizing centers (8). TACC 3 , a member of the TACC domain family, was first identified as a microtubule-binding protein (9). It has been reported that the depletion of TACC3 causes growth retardation and embryonic mortality in mice due to increased apoptosis (10); and TACC3 promotes axon elongation and regulates microtubule plus end dynamics in multiple embryonic cell types (11). In addition, several studies have demonstrated that TACC 3 can promote cell proliferation, metastasis and invasion, and regulate cell cycle progression and differentiation in a variety of cancer cells (12-14). However, the role of TACC 3 in trophoblast function during placental development remains to be fully elucidated. The present study aimed to determine the expression and function of TACC 3 in human placentas and to examine the underlying mechanisms. The results demonstrated that TACC 3 induced trophoblast cell growth, migration and invasion by activation of the phosphoinositide 3-kinase (PI3K)/Akt signaling pathway. TACC 3 may serve as a novel potential target in the treatment of preeclampsia.

\section{Materials and methods}

Tissue specimens. Human placental tissues at 6-8 weeks were obtained from 10 healthy women undergoing cesarean section for non-medical reasons; normal term and preeclamptic 
placentas were collected following cesarean section. The fresh tissue specimens were immediately snap-frozen and stored in liquid nitrogen until use. The protocol for the use of patient samples was approved by the Ethics Committee of the School of Medicine, Zhejiang University (Zhejiang, China) and written informed consent was obtained from each patient.

Cell culture. The immortalized first trimester trophoblast cell line, HTR8/SVneo, was obtained from American Type Culture Collection (Manassas, VA, USA). The cells were cultured in RPMI 1640 medium (Invitrogen; Thermo Fisher Scientific, Inc., Waltham, MA, USA) supplemented with $100 \mathrm{U} / \mathrm{ml}$ penicillin and $100 \mathrm{mg} / \mathrm{ml}$ streptomycin (Sigma-Aldrich; Thermo Fisher Scientific, Inc.) in the presence of $10 \%$ fetal bovine serum (Sigma-Aldrich; Thermo Fisher Scientific, Inc.). The cells were cultured at $37^{\circ} \mathrm{C}$ in a $5 \% \mathrm{CO}_{2}$ incubator.

Small interfering (si)RNA knockdown of TACC3. The siRNA against TACC3 (siRNA-TACC3) and the control (scramble) were purchased from Santa Cruz Biotechnology, Inc. (Santa Cruz, CA, USA). The HTR8/SVneo cells were transfected with either siRNA-TACC3 or scramble siRNA using Lipofectamine $^{\mathrm{TM}} 2000$ (Invitrogen; Thermo Fisher Scientific, Inc.), according to the manufacturer's protocols.

RNA extraction and reverse transcription-quantitative polymerase chain reaction ( $R T-q P C R)$ analysis. Total RNA was extracted from the HTR8/SVneo cells using TRIzol reagent (Abcam, Cambridge, UK) according to the manufacturer's protocol. cDNA was synthesized from the extracted RNA (4 $\mu \mathrm{g})$ using the EasyScript First-Strand cDNA Synthesis SuperMix kit (Invitrogen; Thermo Fisher Scientific, Inc.). The RT-qPCR was performed in a final volume of $10 \mu 1$, containing $5 \mu \mathrm{l}$ of SsoFast ${ }^{\mathrm{TM}}$ EvaGreen Supermix (Bio-Rad Laboratories, Inc., Hercules, CA, USA), $1 \mu \mathrm{l}$ cDNA (1:50 dilution), and $2 \mu \mathrm{l}$ each of the forward and reverse primers $(1 \mathrm{mM})$ with a Bio-Rad iQ5 Quantitative PCR System (Bio-Rad Laboratories, Inc.). PCR amplification was performed using the following primers: TACC3, forward 5'-GAACTGGGGAAGATCATG GA-3' and reverse 5'-CTCTTCGTTCTTGCGGTAGC-3'; and $\beta$-actin, forward 5'-TTAGTTGCGTTACACCCTTTC-3' and reverse 5'-ACCTTCACCGTTCCAGTTT-3'. The PCR conditions consisted of an initial preincubation step at $95^{\circ} \mathrm{C}$ for $30 \mathrm{sec}$, followed by 39 cycles at $95^{\circ} \mathrm{C}$ for $5 \mathrm{sec}$ and $60^{\circ} \mathrm{C}$ for $30 \mathrm{~min}$. The relative mRNA levels were calculated using the $2^{-\Delta \Delta \mathrm{Cq}}$ method (15).

Western blot analysis. Cell lysate was prepared from the HTR8/SVneo cells using lysis buffer (Cell Signaling Technology, Inc., Danvers, MA, USA). Proteins were separated by centrifugation at $6,000 \times \mathrm{g}$ for $15 \mathrm{~min}$ at $4^{\circ} \mathrm{C}$ and the supernatant was collected. Protein concentration was determined by the bicinchoninic acid assay. The proteins (20-30 $\mu \mathrm{g}$ per lane) were separated by 10\% SDS-PAGE and transferred onto a nitrocellulose membrane (EMD Millipore, Boston, MA, USA). The membranes were blocked with 5\% fat-free milk in phosphate-buffered saline with Tween 20 (PBST) at room temperature for $1 \mathrm{~h}$, followed by incubation with primary antibodies (all from Santa Cruz Biotechnology, Inc.) overnight at $4^{\circ} \mathrm{C}$. The primary antibodies were as follows:
Mouse monoclonal anti-TACC3 (1:3,000; cat. no. sc-376883), mouse monoclonal anti-matrix metalloproteinase-2 (MMP-2; 1:1,000; cat. no. sc-13594), mouse monoclonal anti-MMP-9 (1:2,500; cat. no. 21733), mouse monoclonal anti-tissue inhibitor of metalloproteinase 1 (TIMP1; 1:1,500; cat. no. sc-365905), mouse monoclonal anti-TIMP2 (1:1,500; cat. no. 21735), rabbit polyclonal anti-phosphorylated PI3K (1:2,000; cat. no. 293115), mouse monoclonal anti-PI3K (1:2,000; cat. no. 365290), rabbit polyclonal anti-phosphorylated Akt (1:2,000; sc-135650), mouse monoclonal anti-Akt (1:2,000; cat. no. sc-5298) or mouse monoclonal anti-GAPDH (1:1,500; cat. no. sc-365062). The membrane was washed five times with PBST buffer. Goat anti-mouse $(1: 3,000$; cat. no. sc-2302) and goat anti-rabbit (1:3,000; cat. no. sc-2054) horseradish peroxidase-conjugated secondary antibodies were added and incubated at room temperature for $1 \mathrm{~h}$. The protein bands were evaluated using enhanced chemiluminescence (Thermo Fisher Scientific, Inc.).

Cell proliferation assay. The HTR8/SVneo cells were plated in 96-well plates at a density of $\sim 1 \times 10^{4}$ cells/well. The cells were transfected with siRNA-TACC3 or scramble. Following incubation for $24 \mathrm{~h}$ at $37^{\circ} \mathrm{C}, 20 \mathrm{ml}$ of $5 \mathrm{mg} / \mathrm{ml} \mathrm{3-[4,5-dimeth-}$ ylthiazol-2-yl]-2,5-diphenyl tetrazolium bromide (MTT; Sigma-Aldrich; Thermo Fisher Scientific, Inc.) was added to the medium and incubated for $4 \mathrm{~h}$ at $37^{\circ} \mathrm{C}$. The formazan was dissolved in dimethylsulfoxide (150 $\mu \mathrm{l} /$ well; Sigma-Aldrich; Thermo Fisher Scientific, Inc.) for $10 \mathrm{~min}$. The absorbance was measured at $570 \mathrm{~nm}$.

Matrigel invasion assay and Transwell migration assay. Cell invasion was determined by the ability of the cells to cross the $8-\mathrm{mm}$ pores of polycarbonate membranes $(6.5-\mathrm{mm}$ filter; 8-mm pore size; Corning Costar, Inc., Corning, NY, USA). In brief, the HTR8/SVneo cells $\left(1.0 \times 10^{5}\right.$ cells/well) transfected with siRNA-TACC3 or scramble were plated in the upper chambers, and $600 \mu \mathrm{l}$ of RPMI-1640 medium containing $10 \%$ fetal bovine serum was added to the lower chamber. Following incubation for $24 \mathrm{~h}$ under normal conditions, the cells on the upper surface of the base membrane were removed with a sterile cotton swab. The cells, which had transferred to the lower surface of the base membrane were stained with hematoxylin and eosin (Sigma-Aldrich; Thermo Fisher Scientific, Inc.) and the numbers of cells were counted under a Leica microscope (Leica Microsystems GmbH, Wetzlar, Germany). The methods utilized in the Transwell migration assays were similar to those used in the cell invasion assays, with the exception that the inserts were not pre-coated with Matrigel.

Statistical analysis. Data are presented as the mean \pm standard deviation. The differences were analyzed using Student's $t$-test or one-way analysis of variance and Student's $t$-test using SPSS 13.0 (SPSS, Inc., Chicago, IL, USA). P<0.05 was considered to indicate a statistically significant difference.

\section{Results}

Expression of TACC3 is decreased in preeclamptic placentas. The present study first detected the mRNA levels of TACC3 in the preeclamptic placentas using RT-qPCR. As shown in 
A

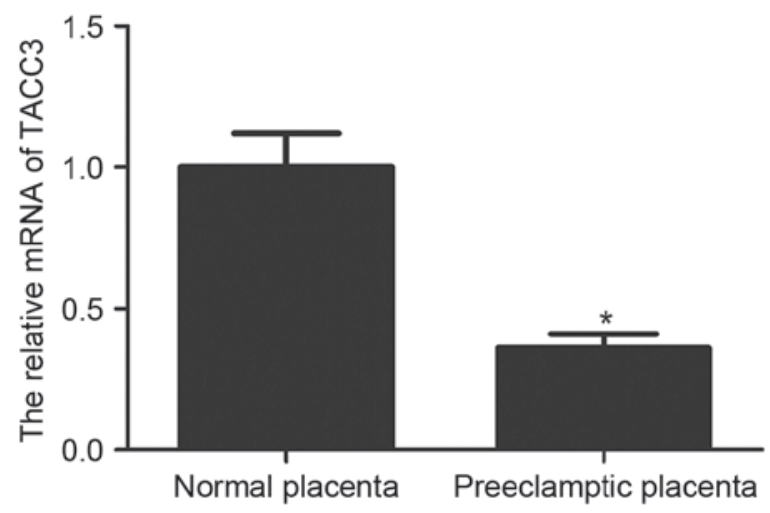

B

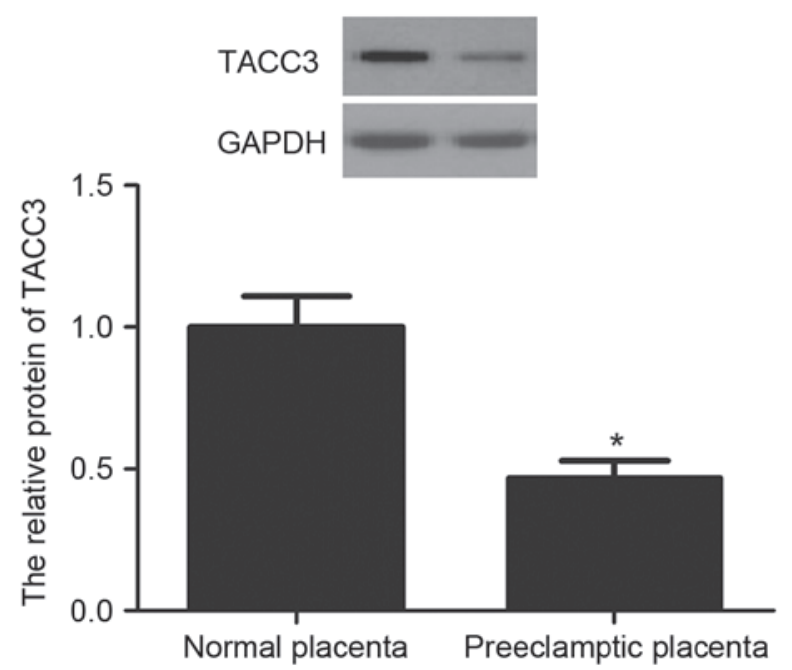

Figure 1. Expression of TACC 3 is decreased in preeclamptic placentas. (A) mRNA expression levels of TACC 3 were analyzed using reverse transcription-quantitative polymerase chain reaction analysis. The mRNA levels of TACC 3 in preeclamptic placentas were significantly lower, compared with those in normal placentas. (B) Representative western blot image and quantification of TACC 3 protein in normal and preeclamptic placentas. Each experiment was repeated three times. Data are presented as the mean \pm standard deviation of triplicate experiments. "P<0.05. TACC 3 , transforming acidic coiled-coil protein 3 .

Fig. 1A, the mRNA levels of TACC3 in the preeclamptic placentas were markedly lower, compared with those in the normal placentas. Western blot analysis showed that the protein levels of TACC 3 were also significantly lower in the preeclampsia placentas, compared with the control group (Fig. 1B). These results suggested that the expression of TACC 3 was reduced in preeclamptic placentas.

Silencing TACC3 significantly inhibits the proliferation of HTR8/SVneo cells. To examine the functional roles of TACC3 in the human placenta, HTR8/SVneo cells were transfected with siRNA-TACC3 or scramble. As shown in Fig. 2A, the mRNA expression of TACC 3 was significantly decreased in the HTR8/SVneo cells transfected with siRNA-TACC3, compared with the scramble group. The knockdown of TACC 3 also reduced the protein expression of TACC 3 (Fig. 2B). An MTT assay was then used to measure the effect of TACC3 on HTR8/SVneo cell proliferation. As shown in Fig. 2C, knockdown of TACC3 significantly inhibited the
A

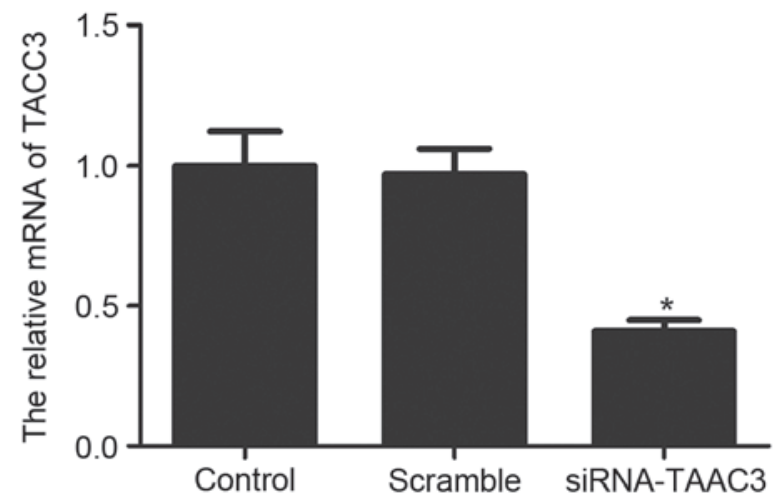

B TACC3

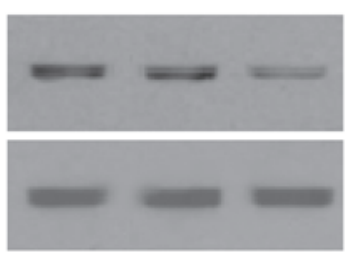

GAPDH

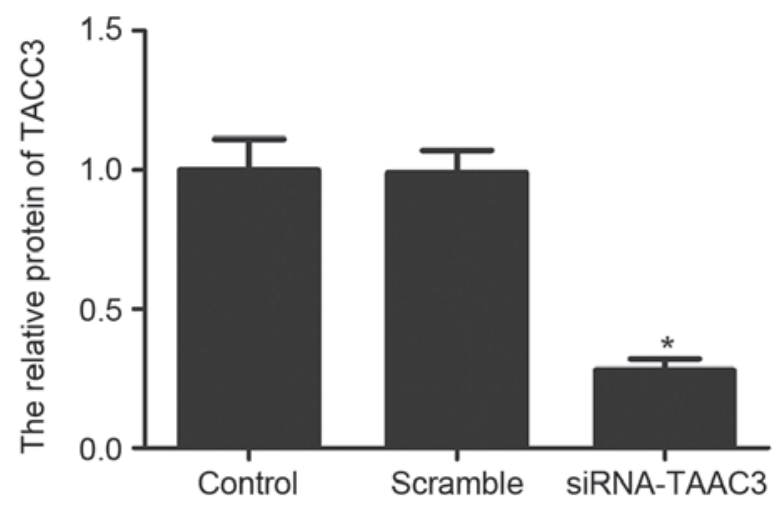

C

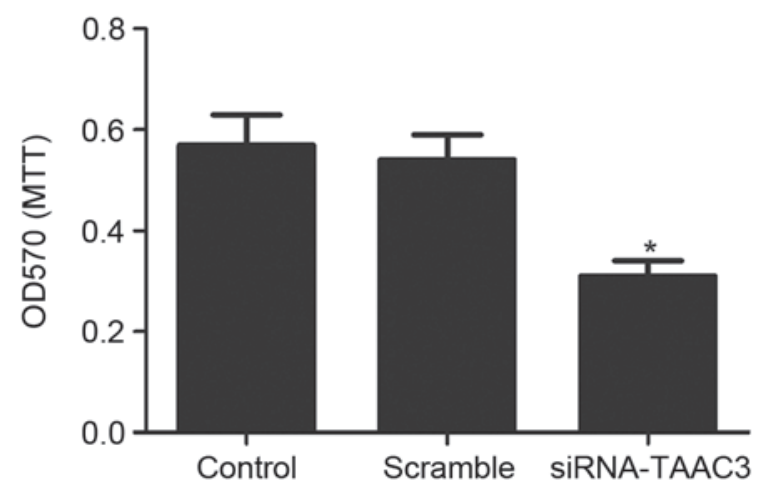

Figure 2. Knockdown of TACC3 inhibits HTR8/SVneo cell proliferation. (A) Reverse transcription-quantitative polymerase chain reaction analysis of mRNA expression levels of TACC3 in HTR8/SVneo cells transfected with siRNA-TACC3 or scramble. (B) Western blot analysis of the protein expression levels of TACC3 in HTR8/SVneo cells transfected with siRNA-TACC3 or scramble. GAPDH was used as an internal control. (C) Proliferation was analyzed using an MTT assay. Knockdown of TACC3 significantly inhibited HTR8/SVneo cell proliferation. Each experiment was repeated three times. Data are presented as the mean \pm standard deviation of triplicate experiments. "P<0.05. TACC3, transforming acidic coiled-coil protein 3; siRNA, small interfering RNA; MTT, 3-[4,5-dimethylthiazol-2-yl]-2,5-diphenyl tetrazolium bromide; $\mathrm{OD}$, optical density. 
A

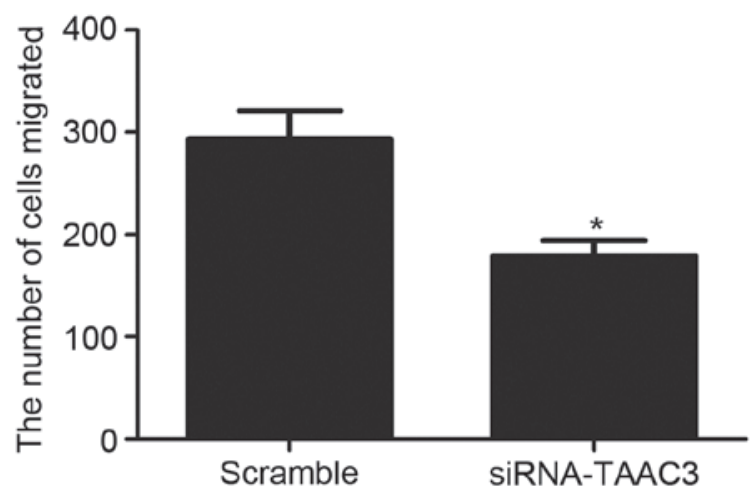

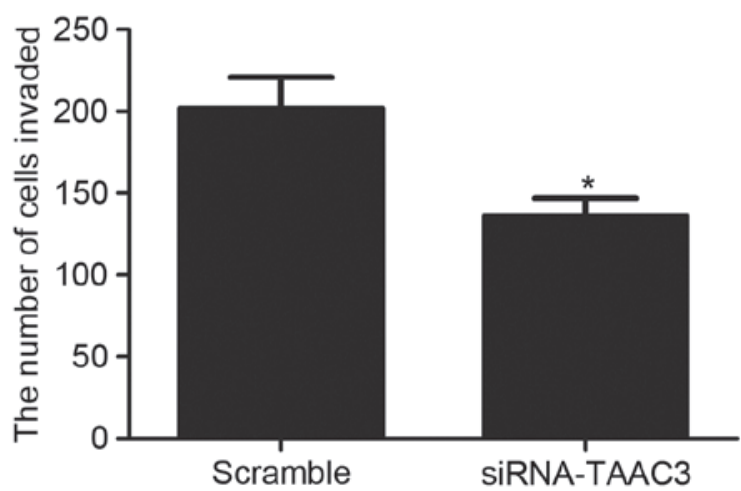

Figure 3. Knockdown of TACC3 inhibits HTR8/SVneo cell migration and invasion. HTR8/SVneo cells were transfected with siRNA-TACC3 or scramble for $24 \mathrm{~h}$. (A) Transwell assay showed that TACC3 knockdown inhibited HTR8/SVneo cell migration, compared with the control. (B) Matrigel invasion assay showed that TACC3 knockdown inhibited HTR8/SVneo cell invasion, compared with the control. Each experiment was repeated three times. Data are presented as the mean \pm standard deviation of triplicate experiments. ${ }^{*} \mathrm{P}<0.05$. TACC 3 , transforming acidic coiled-coil protein 3 ; siRNA, small interfering RNA.

A

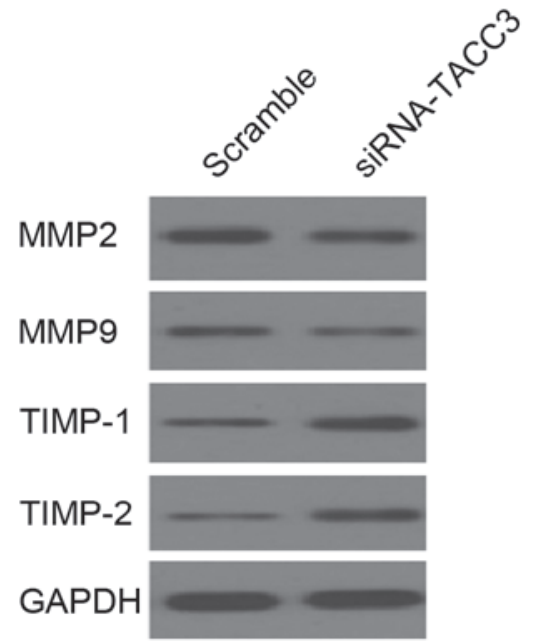

B

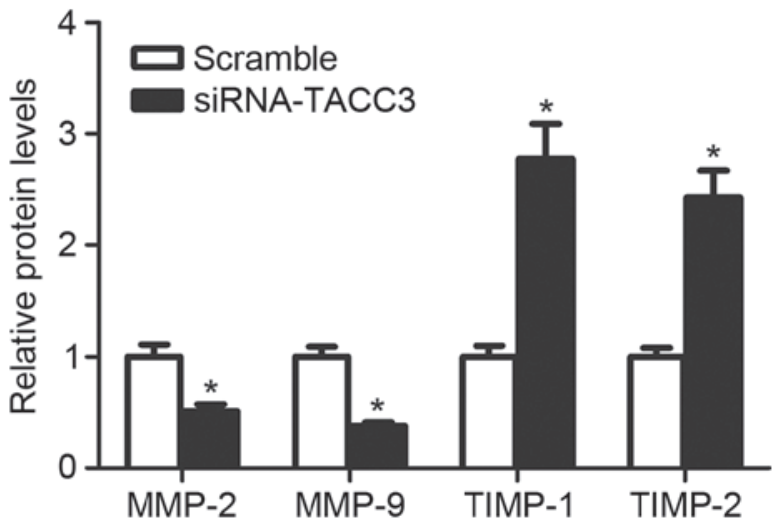

Figure 4. Effects of TACC3 on the expression levels of MMP2/9 and TIMP1/2 in HTR-8/SVneo cells. (A) HTR8/SVneo cells were transfected with siRNA-TACC 3 or scramble for $24 \mathrm{~h}$, and protein expression levels of MMP2/9 and TIMP1/2 were analyzed by western blot analysis. (B) Intensity of expression, compared with the control. Data are presented as the mean \pm standard deviation of triplicate experiments. ${ }^{*} \mathrm{P}<0.05$. TACC 3 , transforming acidic coiled-coil protein 3; siRNA, small interfering RNA; MMP, matrix metalloproteinase; TIMP, tissue inhibitor of metalloproteinase.

A

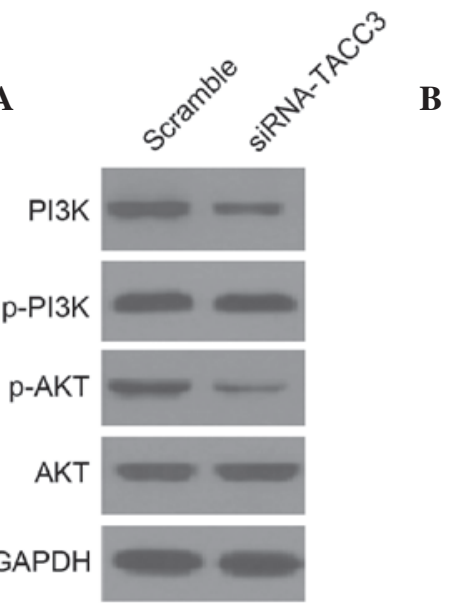

B

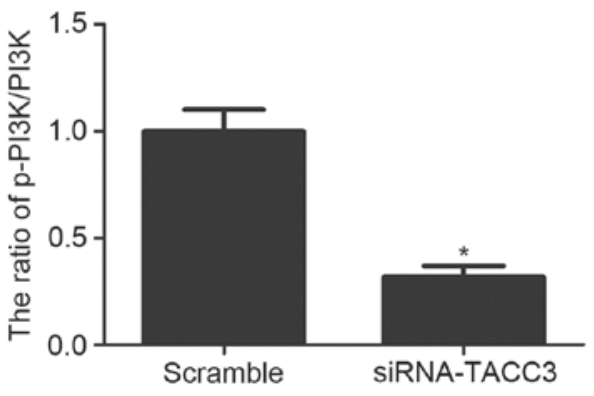

C

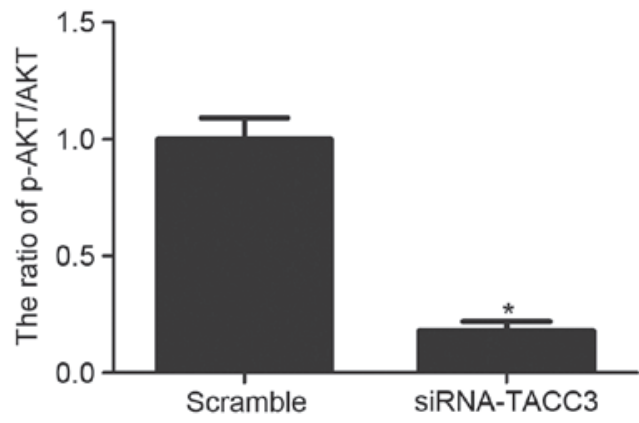

Figure 5. Effect of TACC3 on the PI3K/Akt signaling pathway. (A) HTR8/SVneo cells were transfected with siRNA-TACC3 or scramble for 24 h, and the expression levels of p-PI3K, PI3K, p-Akt and Akt were analyzed using western blot analysis. Intensity of the expression of (B) p-PI3K and (C) p-Akt, compared with the control. Each experiment was repeated three times. Data are presented as the mean \pm standard deviation of triplicate experiments. "P<0.05. TACC3, transforming acidic coiled-coil protein 3; siRNA, small interfering RNA; PI3K, phosphoinositide 3-kinase; p-, phosphorylated. 
proliferation of the HTR8/SVneo cells, compared with the scramble group.

Silencing TACC3 significantly inhibits the migration and invasion of HTR8/SVneo cells. The migration and invasion of trophoblast cells is important in the process of preeclampsia. Therefore, the present study investigated the effect of TACC3 on HTR8/SVneo cell migration and invasion. As shown in Fig. 3, compared with the scramble group, knockdown of TACC3 significantly decreased the percentage of cells that migrated (Fig. 3A) or invaded (Fig. 3B) to the other side of the filter.

Silencing TACC3 reduces the expression of MMP2/9 and increases the expression of TIMP1/2 in HTR8/SVneo cells. It has been reported that MMP2 and MMP9 are critical in trophoblast invasion by degrading the extracellular matrix (ECM) (16). Therefore, the present study determined the effect of TACC3 on the expression of MMPs in HTR8/SVneo cells. As shown in Fig. 4A and B, compared with the scramble group, knockdown of TACC 3 significantly reduced the expression levels of MMP2 and MMP9. By contrast, the knockdown of TACC 3 significantly increased the expression levels of TIMP1 and TIMP2 in the HTR8/SVneo cells.

Silencing TACC3 reduces the phosphorylation levels of PI3K and Akt. The PI3K/Akt pathway is one of the major signaling pathways associated with trophoblast invasion (17). To examine the involvement of the signaling pathway in TACC3-induced HTR8/SVneo cell invasion, the present study evaluated the effects of TACC3 on the PI3K/Akt signaling pathway. As shown in Fig. 5A-C), compared with the scramble group, the knockdown of TACC3 significantly reduced the levels of phosphorylated PI3K and Akt in the HTR8/SVneo cells.

\section{Discussion}

The present study is the first, to the best of our knowledge, to demonstrate the expression and function of TACC 3 in human early placental tissues. The results showed that the expression of TACC3 was downregulated in preeclamptic placentas, compared with normal placentas. The knockdown of TACC3 significantly inhibited HTR8/SVneo cell proliferation, migration and invasion, and inhibited the expression of MMPs. In addition, the knockdown of TACC3 significantly reduced the levels of phosphorylated PI3K and Akt in the HTR8/SVneo cells.

Trophoblast cells have high rates of cell proliferation, lack cell-contact inhibition, and are able to evade effectors of the immune system, particularly during the first trimester of pregnancy (18). For trophoblast cells, invasion can ensure that agents can enter the uterine stroma and gain access to the maternal circulation. Increasing evidence indicates that the deficient migration and shallow invasion of trophoblasts may lead to preeclampsia (19-21). In addition, the invasion/migration properties and the regulatory mechanisms are similar between trophoblasts and malignant cells (22). In the present study, the expression and role of TACC 3 in the progression of preeclampsia were investigated, and it was observed that knockdown of TACC3 significantly inhibited HTR8/SVneo cell proliferation, migration and invasion. These results are consistent with the previously reported role of TACC 3 in human malignancies (13), and suggest that TACC3 may be important in the progression of preeclampsia.

MMPs are a family of zinc-dependent endopeptidases involved in the degradation of the majority of the proteins of the ECM (23). Previous studies have reported that MMPs are important in the process of trophoblast invasion $(16,24,25)$. It has also been reported that MMP-2 is predominantly expressed in extravillous trophoblasts in the placenta during the first trimester (26). In addition, TIMPs have the ability to inhibit the activity of MMPs in the extracellular space by binding specifically to active MMPs (27). In the present study, it was observed that knockdown of TACC3 significantly inhibited the expression of MMP-2 and MMP-9, and increased the expression of TIMP1/2. These results suggested that siRNA-TACC3 may contribute to the downregulation of the expression of MMP-2 and MMP-9, which may lead to reduced HTR8/SVneo cell migration and invasion.

Multiple signals have been demonstrated to be important in trophoblast growth and invasion (28-30). Among these, the PI3K/Akt signaling pathway is a critical pathway mediating the growth-factor-dependent regulation of trophoblast growth and invasion (31). Ha et al (14) reported that TACC3 promotes epithelial-mesenchymal transition through activation of the PI3K/Akt and extracellular signal-regulated kinase signaling pathways in tumor cells (14). To further clarify the underlying mechanism involved in TACC3-inhibited HTR8/SVneo cell growth and invasion, the present study examined the levels of phosphorylated PI3K and Akt following siRNA-TACC3 transfection. It was observed that the knockdown of TACC3 significantly reduced the levels of phosphorylated PI3K and Akt in the HTR8/SVneo cells. These results suggested that siRNA-TACC3 inhibited the invasion and downregulated the expression levels of MMPs in the HTR8/SVneo cells through suppression of the PI3K/Akt signaling pathway.

Taken together, the results of the present study demonstrated that the knockdown of TACC3 inhibited the migration and invasion of HTR8/SVneo cells through suppression of the PI3K/Akt signaling pathway. Therefore, TACC3 may serve as a novel potential target in the treatment of preeclampsia.

\section{References}

1. Kanasaki K and Kalluri R: The biology of preeclampsia. Kidney Int 76: 831-837, 2009.

2. Redline RW and Patterson P: Pre-eclampsia is associated with an excess of proliferative immature intermediate trophoblast. Hum Pathol 26: 594-600, 1995.

3. Fisher SJ: The placental problem: Linking abnormal cytotrophoblast differentiation to the maternal symptoms of preeclampsia. Reprod Biol Endocrinol 2: 53, 2004.

4. Cui Y, Wang W, Dong N, Lou J, Srinivasan DK, Cheng W, Huang X, Liu M, Fang C, Peng J, et al: Role of corin in trophoblast invasion and uterine spiral artery remodelling in pregnancy. Nature 484: 246-250, 2012

5. Myatt L: Role of placenta in preeclampsia. Endocrine 19: 103-111, 2002.

6. O'Brien LL, Albee AJ, Liu L, Tao W, Dobrzyn P, Lizarraga SB and Wiese C: The Xenopus TACC homologue, maskin, functions in mitotic spindle assembly. Mol Biol Cell 16: 2836-2847, 2005.

7. Peset I and Vernos I: The TACC proteins: TACC-ling microtubule dynamics and centrosome function. Trends Cell Biol 18: 379-388, 2008 
8. Gergely F, Karlsson C, Still I, Cowell J, Kilmartin J and Raff JW: The TACC domain identifies a family of centrosomal proteins that can interact with microtubules. Proc Natl Acad Sci USA 97: 14352-14357, 2000.

9. Groisman I, Huang YS, Mendez R, Cao Q, Theurkauf W and Richter JD: CPEB, maskin, and cyclin B1 mRNA at the mitotic apparatus: Implications for local translational control of cell division. Cell 103: 435-447, 2000.

10. Piekorz RP, Hoffmeyer A, Duntsch CD, McKay C, Nakajima H, Sexl V, Snyder L, Rehg J and Ihle JN: The centrosomal protein TACC 3 is essential for hematopoietic stem cell function and genetically interfaces with p53-regulated apoptosis. EMBO J 21: 653-664, 2002

11. Nwagbara BU, Faris AE, Bearce EA, Erdogan B, Ebbert PT, Evans MF, Rutherford EL, Enzenbacher TB and Lowery LA: TACC 3 is a microtubule plus end-tracking protein that promotes axon elongation and also regulates microtubule plus end dynamics in multiple embryonic cell types. Mol Biol Cell 25: 3350-3362, 2014

12. Huang ZL, Lin ZR, Xiao YR, Cao X, Zhu LC, Zeng MS, Zhong Q and Wen ZS: High expression of TACC3 in esophageal squamous cell carcinoma correlates with poor prognosis. Oncotarget 6: 6850-6861, 2015.

13. Ha GH, Kim JL and Breuer EK: TACC3 is essential for EGF-mediated EMT in cervical cancer. PLoS One 8: e70353, 2013.

14. Ha GH, Park JS and Breuer EK: TACC3 promotes epithelial-mesenchymal transition (EMT) through the activation of PI3K/Akt and ERK signaling pathways. Cancer Lett 332: 63-73, 2013.

15. Livak KJ and Schmittgen TD: Analysis of relative gene expression data using real-time quantitative PCR and the 2(-Delta Delta C(T)) method. Methods 25: 402-408, 2001.

16. Cohen M, Meisser A and Bischof P: Metalloproteinases and human placental invasiveness. Placenta 27: 783-793, 2006.

17. Qiu Q, Yang M, Tsang BK and Gruslin A: Both mitogen-activated protein kinase and phosphatidylinositol 3-kinase signalling are required in epidermal growth factor-induced human trophoblast migration. Mol Hum Reprod 10: 677-684, 2004.

18. Ferretti C, Bruni L, Dangles-Marie V, Pecking A and Bellet D: Molecular circuits shared by placental and cancer cells and their implications in the proliferative, invasive and migratory capacities of trophoblasts. Hum Reprod Update 13: 121-141, 2007.
19. Kaufmann P, Black S and Huppertz B: Endovascular trophoblast invasion: Implications for the pathogenesis of intrauterine growth retardation and preeclampsia. Biol Reprod 69: 1-7, 2003.

20. Davison JM,Homuth V, Jeyabalan A, Conrad KP, Karumanchi SA, Quaggin S, Dechend R and Luft FC: New aspects in the pathophysiology of preeclampsia. J Am Soc Nephrol 15: 2440-2448, 2004.

21. Pijnenborg R, Vercruysse L and Hanssens M: Fetal-maternal conflict, trophoblast invasion, preeclampsia, and the red queen. Hypertens Pregnancy 27: 183-196, 2008.

22. Mullen CA: Review: Analogies between trophoblastic and malignant cells. Am J Reprod Immunol 39: 41-49, 1998.

23. Westermarck J and Kähäri VM: Regulation of matrix metalloproteinase expression in tumor invasion. FASEB J 13: 781-792, 1999.

24. Staun-Ram E, Goldman S, Gabarin D and Shalev E: Expression and importance of matrix metalloproteinase 2 and 9 (MMP-2 and -9) in human trophoblast invasion. Reprod Biol Endocrinol 2: $59,2004$.

25. Hulboy DL, Rudolph LA and Matrisian LM: Matrix metalloproteinases as mediators of reproductive function. Mol Hum Reprod 3: 27-45, 1997.

26. Isaka K, Usuda S, Ito H, Sagawa Y, Nakamura H, Nishi $H$, Suzuki Y, Li Y and Takayama M: Expression and activity of matrix metalloproteinase 2 and 9 in human trophoblasts. Placenta 24: 53-64, 2003.

27. Nagase H, Visse R and Murphy G: Structure and function of matrix metalloproteinases and TIMPs. Cardiovasc Res 69: 562-573, 2006

28. Prast J, Saleh L, Husslein H, Sonderegger S, Helmer H and Knöfler M: Human chorionic gonadotropin stimulates trophoblast invasion through extracellularly regulated kinase and AKT signaling. Endocrinology 149: 979-987, 2008.

29. Chakraborty C, Gleeson LM, McKinnon T and Lala PK: Regulation of human trophoblast migration and invasiveness. Can J Physiol Pharmacol 80: 116-124, 2002.

30. Fitzgerald JS, Poehlmann TG, Schleussner E and Markert UR: Trophoblast invasion: The role of intracellular cytokine signalling via signal transducer and activator of transcription 3 (STAT3). Hum Reprod Update 14: 335-344, 2008.

31. Knöfler M and Pollheimer J: IFPA Award in Placentology lecture: Molecular regulation of human trophoblast invasion. Placenta 33 (Suppl): S55-S62, 2012. 